\title{
Red meat consumption is associated with an increased overall cancer risk: a prospective cohort study in Korea
}

\author{
Gyung-Ah Wie ${ }^{1 *}$, Yeong-Ah Cho ${ }^{1}$, Hyun-hee Kang ${ }^{1}$, Kyoung-A Ryu ${ }^{1}$, Min-Kyoung Yoo ${ }^{1}$, Young-A Kim ${ }^{2}$, \\ Kyu-Won Jung ${ }^{3}$, Jeongseon Kim ${ }^{4}$, Joo-Hyuk Lee ${ }^{5}$ and Hyojee Joung ${ }^{6 * *}$ \\ ${ }^{1}$ Department of Clinical Nutrition, Research Institute and Hospital, National Cancer Center, 323 Ilsan-ro, \\ Ilsandong-gu, Goyang-si, Gyeonggi-do 410-769, Republic of Korea \\ ${ }^{2}$ Department of Food and Nutrition, College of Human Ecology, Inha University, Incheon, Republic of Korea \\ ${ }^{3}$ The Korean Central Cancer Registry, National Cancer Center, Goyang-si, Gyeonggi-do, Republic of Korea \\ ${ }^{4}$ Division of Cancer Epidemiology and Management, National Cancer Center, Goyang-si, Gyeonggi-do, Republic of Korea \\ ${ }^{5}$ Department of Radiology, Research Institute and Hospital, National Cancer Center, Goyang-si, Gyeonggi-do, \\ Republic of Korea \\ ${ }^{6}$ Graduate School of Public Health and Institute of Health and Environment, Seoul National University, 599 Gwanak-ro, \\ Gwanak-gu, Seoul 151-742, Republic of Korea
}

(Submitted 8 August 2013 - Final revision received 27 February 2014 - Accepted 3 March 2014 - First published online 28 April 2014)

\begin{abstract}
Cancer is a leading cause of death, and the dietary pattern in Korea is changing rapidly from a traditional Korean diet to a Westernised diet. In the present study, we investigated the effects of dietary factors on cancer risk with a prospective cohort study. Among 26815 individuals who participated in cancer screening examinations from September 2004 to December 2008, 8024 subjects who completed a selfadministered questionnaire concerning demographic and lifestyle factors, and a $3 \mathrm{~d}$ food record were selected. As of September 2013 , 387 cancer cases were identified from the National Cancer Registry System, and the remaining individuals were included in the control group. The hazard ratio (HR) of cancer for the subjects older than or equal to 50 years of age was higher (HR 1.80, 95\% CI 1.41, 2.31; $P<0.0001)$ than that for the other subjects. Red meat consumption, Na intake and obesity (BMI $\left.\geq 25 \mathrm{~kg} / \mathrm{m}^{2}\right)$ were positively associated with overall cancer incidence in men (HR 1.41, $95 \%$ CI 1.02, 1.94; $P=0 \cdot 0382$ ), gastric cancer (HR 2.34, 95\% CI 1.06, 5.19; $P=0 \cdot 0365$ ) and thyroid cancer (HR 1.56, $95 \%$ CI 1.05, 2.31; $P=0.0270$ ), respectively. Participants who had at least three dietary risk factors among the high intakes of red meat and $\mathrm{Na}$, low intakes of vegetables and fruits, and obesity suggested by the World Cancer Research Fund/ American Institute for Cancer Research at baseline tended to have a higher risk of cancer than the others (HR 1.26, 95\% CI 0.99, 1.60; $P=0.0653)$. In summary, high intakes of red meat and Na were significant risk factors of cancer among Koreans.
\end{abstract}

Key words: Cancer risk: Red meat: Sodium: Prospective cohort studies

In Korea, cancer has been the leading cause of death since 1983 , and the overall incidence rate has increased by $3.3 \%$ per year (1.5\% in males and 5.3\% in females) from 1999 to $2010^{(1,2)}$. The incidence rates of prostate, breast and colorectal cancers have increased, whereas the rates of cervical uterine and liver cancers have decreased ${ }^{(2)}$. Many epidemiological studies have suggested that the risk of these cancers is associated with Westernised lifestyles ${ }^{(3-6)}$

According to the report by 2007 World Cancer Research Fund/American Institute for Cancer Research (WCRF/AICR), lifestyle factors such as diet, physical activity, alcohol consumption and cigarette smoking are the major risk factors for cancer ${ }^{(3)}$. Dietary risk factors, including high intakes of red meat and $\mathrm{Na}$, low intakes of vegetables and fruits, and obesity, have been thought to account for approximately $30 \%$ of cancer incidence in Western countries ${ }^{(7-24)}$.

Korea has experienced a rapid and unique nutrition transition due to rapid economic growth and the introduction of Western culture over the last several decades ${ }^{(25-27)}$.

The dietary pattern has shifted from a traditional Korean diet that is mostly based on rice and vegetables to a Westernised diet composed of mainly meat and their products ${ }^{(25-29)}$.

Abbreviations: AICR, American Institute for Cancer Research; HR, hazard ratio; WCRF, World Cancer Research Fund.

*Corresponding authors: G.-A. Wie, fax +82 31920 0269, email gawie@ncc.re.kr; H. Joung, fax +82 28832832 , email hjjoung@snu.ac.kr 
Although intakes of fruits and vegetables among Koreans have been increasing, intake of salty fermented vegetables including kimchi remains to be high ${ }^{(30)}$.

However, most epidemiological studies about the associations between dietary factors and cancer risk among Koreans are restricted to case-control study designs or specific cancer sites or selected regions or several nutrients ${ }^{(15,16,31-34)}$. Scientific evidence for the association between dietary factors and cancer risk among Koreans should be derived from prospective cohort studies or clinical trials to develop dietary guidelines for cancer prevention, because the epidemiological characteristics of cancer risk factors among Koreans may not be the same as in the people of Western societies.

Therefore, the present study was designed to investigate the association between dietary factors and cancer risk among Koreans using the data from the Cancer Screening Examination Cohort of the National Cancer Center of Korea.

\section{Experimental methods}

\section{Study design and population}

Of the 26815 individuals who participated in the Cancer Screening Examination Cohort of the National Cancer Center of Korea from 1 September 2004 to 31 December 2008, 8179 participants who completed a self-administered questionnaire concerning demographic factors, medical history and behavioural factors, and a $3 \mathrm{~d}$ food record were selected for the present study. The details of the study population are shown in Fig. 1.

We excluded the participants with any history of cancer at baseline $(n 79)$ or who reported an implausible daily energy intake $(<2929$ or $>20920 \mathrm{~kJ}(<700$ or $>5000 \mathrm{kcal}) ; n 29)$ Cancer incidence among all participants after baseline was confirmed using the Korean Central Cancer Registry and/or the Electronic Medical Record of the National Cancer Center. As of 24 September 2013, 387 subjects were identified as cancer patients according to the International Classification of Diseases-10 Codes C00-C99, with the exception of Code $\mathrm{D}$ ( $n$ 47), and the remaining were assigned to the control group $(n 7637)^{(35)}$. The number of subjects included in the final analysis was 8024 .

Written informed consent was obtained from all subjects, and the study protocol was approved by the Institutional Review Board of the National Cancer Center (NCCNCS-09-274).

\section{Dietary and lifestyle data collection}

All participants were asked to complete a self-administered questionnaire concerning their demographic factors (e.g. age, education, occupation, household income and marital status), behavioural factors (e.g. cigarette smoking, alcohol intake and regular exercise) and personal medical history.

For each subject, height and weight were measured using InBody 3.0 (Biospace), and BMI was calculated by dividing weight $(\mathrm{kg})$ by the square of height $\left(\mathrm{m}^{2}\right)$. According to the criteria of the WHO Asia-Pacific Region, those subjects with a $\mathrm{BMI} \geq 25 \mathrm{~kg} / \mathrm{m}^{2}$ were classified as obese ${ }^{(36,37)}$.

Data for sex, age, height and weight, and cancer site and diagnosis time were ascertained using the clinical database of the Korean National Cancer Center.

Dietary intake data were obtained from a $3 \mathrm{~d}$ food record. The participants were asked to complete a food record for 2 weekdays and 1 weekend day within a week. To increase
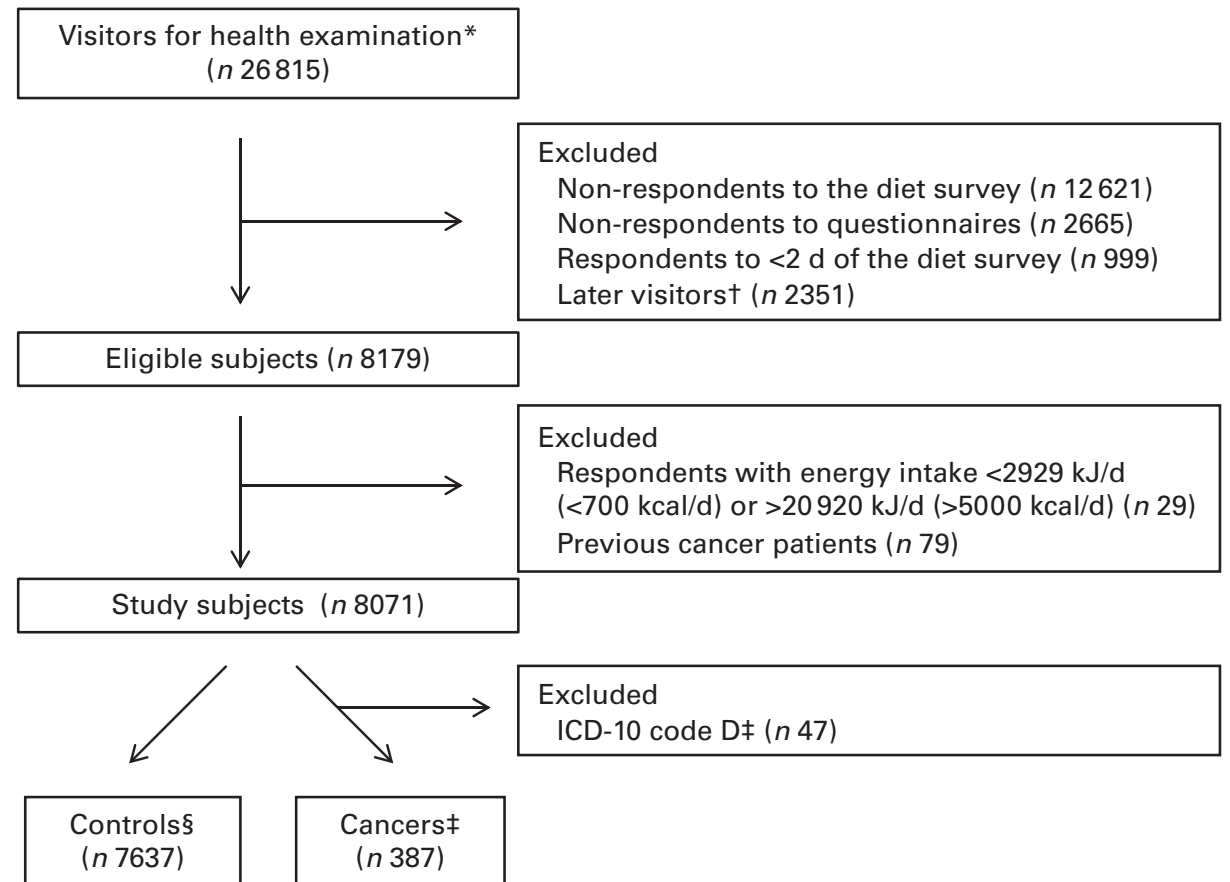

Fig. 1. Inclusion and exclusion criteria for the study subjects. ${ }^{*}$ Visitors for the health examination survey from 1 September 2004 to 31 December 2008 $\dagger$ Excluded the data from the first visit if the participants visited more than twice. $\ddagger$ Newly detected cancer according to the WHO International Classification of Diseases (ICD)-10 as of 24 September 2013. §No detected cancer or previous cancer history as of 24 September 2013. 
the accuracy of food records, trained registered dietitians confirmed all records written by subjects with face-to-face interviews. The daily intake of energy, nutrients, food and food groups for each subject were calculated using CAN-Pro 3.0 (Korean Nutrition Society).

\section{Dietary risk factors}

We investigated four dietary risk factors (high intakes of red meat and $\mathrm{Na}$, low intakes of vegetables and fruits, and obesity) based on a priori knowledge of risk factors for overall cancer incidence. Because no dietary guidelines for cancer prevention are available in Korea, the recommendations for the intakes of red meat, fruits and vegetables, and $\mathrm{Na}$ provided by the WCRF/AICR (2007) were adopted as reference intakes for cancer-related dietary factors ${ }^{(3)}$. The WCRF/AICR recommended that the consumption of red meat and $\mathrm{Na}$ should be limited to $<300 \mathrm{~g} /$ week $(43 \mathrm{~g} / \mathrm{d}$ ) and $<4 \mathrm{~g} / \mathrm{d}$, respectively, and intakes of vegetables and fruits should be at least $600 \mathrm{~g} / \mathrm{d}$. In the present study, red meat referred to beef, pork, lamb and goat, and processed meats. Processed meats included meats contained in processed foods such as bacon, ham and sausage, and the internal organs (offal, such as the brain, liver, heart, intestines and tongue). Vegetables were considered as the edible parts of plants, which generally includes fungi, and fruits were considered as the seed-containing part of plants. Vegetables and fruits preserved by salting and/or pickling (e.g. kimchi in Korea) were not included. Na intake was estimated from $3 \mathrm{~d}$ food records by Can-Pro 3.0 (Korean Nutrition Society).

Obesity was considered as a dietary risk factor because this condition reflects excessive energy intake. Each factor was dichotomised into two categories: no risk ( 0 points) and risk (1 point). All participants scored 1 point for each of the following dietary risk factors at baseline: $\geq 43 \mathrm{~g}$ red meat $/ \mathrm{d} ;<600 \mathrm{~g}$ vegetables and fruits/d; $\geq 4 \mathrm{~g} \mathrm{Na} / \mathrm{d}$; obesity $\left(\mathrm{BMI} \geq 25 \mathrm{~kg} / \mathrm{m}^{2}\right)$. We determined the number of dietary risk factors per participant by summing the scores for each of the four dietary risk factors; consequently, the number of dietary risk factors ranged from 0 to 4 points.

\section{Statistical analyses}

The characteristics of the study population were described as the number and percentage of subjects in the cancer and control groups, and the $\chi^{2}$ test and generalised linear regression analysis were used to compare the distributions of subjects and mean differences between the two groups, respectively. The hazard ratio (HR) and 95\% CI were estimated using the Cox proportional hazards regression model. The multivariate model was adjusted for age, sex, energy intake, BMI, physical activity (yes or no), smoking (yes or no), alcohol use (yes or no), income (three categories), education (three categories) and marital status (three categories). In addition, HR in several subgroups were analysed: sex (male, female); age ( $<50$ years, $\geq 50$ years); cancer sites (thyroid, gastric and colon cancers).
All statistical analyses were performed using SAS software (version 9.1.3; SAS Institute, Inc.). Statistical significance was set at $P<0.05$.

\section{Results}

During 54027 person-years of follow-up (median $7 \cdot 0$ years), 387 subjects were diagnosed with cancer. The detailed characteristics of the study subjects are shown in Table 1.In comparison with the controls, cancer subjects were older $(P<0 \cdot 0001)$, had lower levels of income $(P=0.0135)$ and education $(P=0.0031)$, and were less likely to be married $(P=0 \cdot 0368)$. There were no significant differences in smoking or physical activity between the cancer and control groups. The total food intake of the control group was higher than that of the cancer group $(P=0.0237)$. The percentage of participants with more than three dietary risk factors in the cancer group was higher than that of the control group $(P=0 \cdot 0387)$.

The HR of cancer according to dietary risk factors is presented in Table 2 .

Obesity (BMI $\geq 25 \mathrm{~kg} / \mathrm{m}^{2}$ ) was significantly associated with overall cancer risk (crude HR 1.24) in total subjects and in women (crude HR 1.60), but the associations were disappeared after adjusting for confounding variables such as age, energy intake and other demographic factors.

The risk of cancer in male was significantly increased among individuals who consumed at least $43 \mathrm{~g}$ red meat $/ \mathrm{d}$ (or $300 \mathrm{~g} /$ week) compared with those who ate less than $43 \mathrm{~g} / \mathrm{d}$ (or $300 \mathrm{~g} /$ week) (HR 1.41, 95\% CI 1.02, 1.94; $P=0.0382$ ) after adjusting for confounding variables.

There were no significant differences in cancer incidence for the intakes of vegetables and fruits and $\mathrm{Na}$ between the cancer and control groups.

The subjects with more than three dietary risk factors among obesity and high intakes of red meat and $\mathrm{Na}$, and low intakes of fruits and vegetables had increased cancer incidence compared with those with two dietary risk factors or less among the total population (crude HR 1.24, 95\% CI 1.01, 1.51; $P=0.0372$ ), women (crude HR 1.52, $95 \%$ CI 1.11, 2.09; $P=0 \cdot 0089)$ and those subjects younger than 50 years of age (crude HR 1.44, $95 \%$ CI 1.06, 1.96; $P=0.0216$ ); however, these significant associations were disappeared after adjusting for confounding variables.

The risk of thyroid, gastric and colorectal cancers, which were most prevalent among Koreans ${ }^{(38)}$, according to the status of having dietary risk factors are shown in Table 3.

The risk of thyroid cancer was higher in the obese subjects (BMI $\geq 25 \mathrm{~kg} / \mathrm{m}^{2}$ ) than the normal-weight subjects (HR 1.56 , $95 \%$ CI $1.05,2.31 ; P=0.0270)$, and the risk of gastric cancer was positively associated with age (HR 2.49, 95\% CI 1.24, 4.99; $P=0 \cdot 0103$; data not shown) and Na intake (HR 2.34, 95\% CI $1 \cdot 06,5 \cdot 19 ; P=0 \cdot 0365)$ after adjusting for confounding variables.

While the risk of colorectal cancer was not significantly associated with the dietary risk factors, the risk of gastrointestinal cancers including gastric and colorectal cancers was positively associated with $\mathrm{Na}$ intake (HR 4.28, 95\% CI $2 \cdot 11,8 \cdot 72 ; P<0.0001)$ and the number of dietary risk factors (HR $1.87,95 \%$ CI $1.03,3.37 ; P=0.0386$ ) after adjusting for confounding variables. 
Table 1. Characteristics of the study subjects

(Mean values and standard deviations; number of subjects and percentages)

\begin{tabular}{|c|c|c|c|c|c|c|c|c|c|c|c|c|c|c|}
\hline & \multirow[b]{3}{*}{ Person-years } & \multirow[b]{3}{*}{ Median } & & & \multicolumn{10}{|c|}{ Cancer subjects } \\
\hline & & & \multicolumn{2}{|c|}{$\begin{array}{l}\text { Controls* } \\
(n \text { 7637) }\end{array}$} & \multicolumn{2}{|c|}{$\begin{array}{l}\text { Total cancers* } \\
\quad(n 387)\end{array}$} & \multicolumn{2}{|c|}{$\begin{array}{l}\text { Thyroid cancer } \\
\qquad(n 136)\end{array}$} & \multicolumn{2}{|c|}{$\begin{array}{c}\text { Gastric } \\
\text { cancer }(n 46)\end{array}$} & \multicolumn{2}{|c|}{$\begin{array}{c}\text { Colorectal } \\
\text { cancer ( } n 53)\end{array}$} & \multicolumn{2}{|c|}{$\begin{array}{c}\text { Gastric and } \\
\text { colorectal } \\
\text { cancer }(n 99)\end{array}$} \\
\hline & & & $n$ & $\%$ & $n$ & $\%$ & $n$ & $\%$ & $n$ & $\%$ & $n$ & $\%$ & $n$ & $\%$ \\
\hline \multicolumn{15}{|l|}{ Demographic factors } \\
\hline $\begin{array}{l}\text { Sex, female } \\
\text { Age (years) }\end{array}$ & $24476 \cdot 2$ & $7 \cdot 0$ & 3457 & $45 \cdot 3$ & 165 & $42 \cdot 6$ & 72 & $52 \cdot 9$ & 15 & $32 \cdot 6$ & 30 & $37 \cdot 7$ & 45 & $45 \cdot 5$ \\
\hline Mean & & & \multirow{2}{*}{\multicolumn{2}{|c|}{$\begin{array}{c}48 \cdot 4 \\
9.2\end{array}$}} & \multirow{2}{*}{\multicolumn{2}{|c|}{$\begin{array}{c}52.5 \\
9.6\end{array}$}} & \multirow{2}{*}{\multicolumn{2}{|c|}{$49 \cdot 3$}} & \multirow{2}{*}{\multicolumn{2}{|c|}{$\begin{array}{c}54 \cdot 1 \\
9.1\end{array}$}} & \multirow{2}{*}{\multicolumn{2}{|c|}{$52 \cdot 1$}} & \multirow{2}{*}{\multicolumn{2}{|c|}{$\begin{array}{c}53.0 \\
9.9\end{array}$}} \\
\hline $\mathrm{SD}$ & & & & & & & & & & & & & & \\
\hline$<50$ years & $30287 \cdot 0$ & 6.9 & 4343 & $56 \cdot 9$ & 163 & $42 \cdot 1$ & 78 & $57 \cdot 4$ & 15 & $32 \cdot 6$ & 23 & 43.4 & 38 & 38.4 \\
\hline$\geq 50$ years & $23739 \cdot 7$ & $7 \cdot 1$ & 3294 & $43 \cdot 1$ & 224 & 57.9 & 58 & $42 \cdot 7$ & 31 & 67.4 & 30 & $56 \cdot 6$ & 61 & 61.4 \\
\hline \multicolumn{15}{|l|}{ BMI $\left(\mathrm{kg} / \mathrm{m}^{2}\right)$} \\
\hline Mean & & & \multirow{2}{*}{\multicolumn{2}{|c|}{$\begin{array}{c}23 \cdot 8 \\
2.9\end{array}$}} & \multicolumn{2}{|c|}{$24 \cdot 3$} & \multicolumn{2}{|c|}{$24 \cdot 3$} & \multicolumn{2}{|c|}{$24 \cdot 8$} & & & & \\
\hline SD & & & & & & & & & & & & & & \\
\hline$<25.0 \mathrm{~kg} / \mathrm{m}^{2}$ & $32463 \cdot 1$ & $6 \cdot 9$ & 4598 & $60 \cdot 2$ & 212 & 54.8 & 72 & 52.9 & 20 & 43.5 & 34 & $64 \cdot 2$ & 54 & 45 \\
\hline$\geq 25.0 \mathrm{~kg} / \mathrm{m}^{2}$ & $21613 \cdot 0$ & $7 \cdot 0$ & 3039 & 39.8 & 175 & $45 \cdot 2$ & 64 & $47 \cdot 1$ & 26 & $56 \cdot 5$ & 19 & $35 \cdot 8$ & 54.5 & 45.5 \\
\hline Income & & & & & & & & & & & & & & \\
\hline$\leq 4$ millions/month & $19455 \cdot 4$ & $7 \cdot 1$ & 2703 & 35.4 & 161 & $41 \cdot 6$ & 62 & $45 \cdot 6$ & 19 & $41 \cdot 3$ & 19 & $35 \cdot 8$ & 38 & 14 \\
\hline $400-700$ millions/month & 20972.5 & $7 \cdot 4$ & 2886 & 37.8 & 117 & $30 \cdot 2$ & 39 & $28 \cdot 7$ & 13 & $28 \cdot 3$ & 19 & 35.8 & 38.4 & 14.1 \\
\hline$\geq 700$ millions/month & $8705 \cdot 3$ & $6 \cdot 1$ & 1363 & 17.8 & 68 & $17 \cdot 6$ & 24 & $17 \cdot 6$ & 6 & $13 \cdot 0$ & 8 & $15 \cdot 1$ & 32 & 15 \\
\hline Missing $†$ & $4943 \cdot 0$ & $7 \cdot 1$ & 685 & 9.0 & 41 & $10 \cdot 6$ & 11 & $8 \cdot 1$ & 8 & $17 \cdot 4$ & 7 & $13 \cdot 3$ & $32 \cdot 3$ & $15 \cdot 2$ \\
\hline Education & & & & & & & & & & & & & & \\
\hline$\leq$ Middle school & $6895 \cdot 7$ & $7 \cdot 1$ & 951 & $12 \cdot 4$ & 68 & $17 \cdot 6$ & 25 & $18 \cdot 4$ & 10 & $21 \cdot 7$ & 8 & $15 \cdot 1$ & 18 & 49 \\
\hline High school & $18002 \cdot 0$ & $6 \cdot 8$ & 2570 & 33.7 & 123 & $31 \cdot 8$ & 42 & $30 \cdot 9$ & 12 & $26 \cdot 1$ & 12 & $22 \cdot 6$ & $18 \cdot 2$ & 49.5 \\
\hline$\geq$ College & $27738 \cdot 8$ & $7 \cdot 0$ & 3918 & 51.3 & 178 & $46 \cdot 0$ & 64 & $47 \cdot 0$ & 21 & $45 \cdot 7$ & 28 & $52 \cdot 8$ & 24 & 8 \\
\hline Missingt & 1434.5 & $6 \cdot 9$ & 198 & $2 \cdot 6$ & 18 & 4.6 & 5 & 3.7 & 3 & 6.5 & 5 & 9.5 & $24 \cdot 2$ & $8 \cdot 1$ \\
\hline Marital status & & & & & & & & & & & & & & \\
\hline Unmarried & 1432.0 & $6 \cdot 9$ & 206 & $2 \cdot 7$ & 7 & 1.8 & 3 & $2 \cdot 2$ & 1 & $2 \cdot 2$ & 0 & 0.0 & 1 & 7 \\
\hline Married & $48772 \cdot 1$ & $7 \cdot 0$ & 6883 & $90 \cdot 1$ & 337 & $87 \cdot 1$ & 116 & $85 \cdot 3$ & 40 & $87 \cdot 0$ & 43 & $81 \cdot 1$ & 1.0 & $7 \cdot 1$ \\
\hline Divorced/widowed & $2658 \cdot 2$ & $6 \cdot 6$ & 380 & $5 \cdot 0$ & 27 & $7 \cdot 0$ & 12 & $8 \cdot 8$ & 1 & $2 \cdot 2$ & 6 & $11 \cdot 3$ & 83 & 8 \\
\hline Missingt & $1213 \cdot 8$ & 6.9 & 168 & $2 \cdot 2$ & 16 & $4 \cdot 1$ & 5 & $3 \cdot 7$ & 3 & 8.6 & 4 & $7 \cdot 6$ & $83 \cdot 8$ & $8 \cdot 1$ \\
\hline Behavioural factors & & & & & & & & & & & & & & \\
\hline Smoking & & & & & & & & & & & & & & \\
\hline No & $24940 \cdot 0$ & $6 \cdot 9$ & 3555 & 46.5 & 177 & $45 \cdot 7$ & 75 & $55 \cdot 2$ & 16 & 34.8 & 25 & $47 \cdot 2$ & 41 & 41.4 \\
\hline Current or former & $24279 \cdot 2$ & $6 \cdot 8$ & 3462 & $45 \cdot 3$ & 179 & $46 \cdot 3$ & 52 & $38 \cdot 2$ & 24 & $52 \cdot 2$ & 24 & $45 \cdot \overline{3}$ & 18 & 48.5 \\
\hline Missing† & $4857 \cdot 0$ & $7 \cdot 7$ & 620 & $8 \cdot 2$ & 31 & $8 \cdot 0$ & 9 & $6 \cdot 6$ & 6 & $13 \cdot 0$ & 4 & 7.5 & 10 & $10 \cdot 1$ \\
\hline Alcohol use & & & & & & & & & & & & & & \\
\hline No & 13664.9 & $6 \cdot 9$ & 1944 & 25.5 & 103 & $26 \cdot 6$ & 40 & 29.4 & 10 & $21 \cdot 7$ & 16 & $30 \cdot 2$ & 26 & $26 \cdot 3$ \\
\hline Current or former & 37318.7 & $7 \cdot 0$ & 5262 & 68.9 & 264 & $68 \cdot 2$ & 86 & $63 \cdot 2$ & 35 & $76 \cdot 1$ & 35 & $66 \cdot 0$ & 70 & $70 \cdot 7$ \\
\hline Missing $†$ & $3092 \cdot 6$ & 6.9 & 431 & 5.6 & 20 & $5 \cdot 2$ & 10 & 7.4 & 1 & $2 \cdot 2$ & 2 & 3.8 & 3 & 3.0 \\
\hline Regular physical activity & & & & & & & & & & & & & & \\
\hline Yes & $24002 \cdot 4$ & 7.5 & 3199 & 41.9 & 147 & $38 \cdot 0$ & 45 & $33 \cdot 1$ & 23 & $50 \cdot 0$ & 22 & 41.5 & 45 & 45.4 \\
\hline No & $26108 \cdot 1$ & $6 \cdot 1$ & 3933 & 51.5 & 204 & $52 \cdot 7$ & 78 & $57 \cdot 3$ & 20 & 43.5 & 27 & $50 \cdot 9$ & 47 & 47.5 \\
\hline Missing $†$ & 3965.6 & $7 \cdot 6$ & 505 & 6.6 & 36 & $9 \cdot 3$ & 13 & $9 \cdot 6$ & 3 & 6.5 & 4 & 7.6 & 7 & $7 \cdot 1$ \\
\hline
\end{tabular}




\begin{tabular}{|c|c|c|c|c|c|c|c|c|c|c|c|c|c|c|}
\hline & \multirow[b]{3}{*}{ Person-years } & \multirow[b]{3}{*}{ Median } & & & \multicolumn{10}{|c|}{ Cancer subjects } \\
\hline & & & \multicolumn{2}{|c|}{$\begin{array}{l}\text { Controls* } \\
(n \text { 7637) }\end{array}$} & \multicolumn{2}{|c|}{$\begin{array}{l}\text { Total cancers }{ }^{*} \\
\quad(n 387)\end{array}$} & \multicolumn{2}{|c|}{$\begin{array}{l}\text { Thyroid cancer } \\
\quad(n 136)\end{array}$} & \multicolumn{2}{|c|}{$\begin{array}{c}\text { Gastric } \\
\text { cancer }(n 46)\end{array}$} & \multicolumn{2}{|c|}{$\begin{array}{c}\text { Colorectal } \\
\text { cancer ( } n \text { 53) }\end{array}$} & \multicolumn{2}{|c|}{$\begin{array}{c}\text { Gastric and } \\
\text { colorectal } \\
\text { cancer ( } n \text { 99) }\end{array}$} \\
\hline & & & $n$ & $\%$ & $n$ & $\%$ & $n$ & $\%$ & $n$ & $\%$ & $n$ & $\%$ & $n$ & $\%$ \\
\hline \multicolumn{15}{|c|}{ Dietary factors } \\
\hline \multicolumn{15}{|c|}{ Total food intake $(\mathrm{g} / \mathrm{d})$} \\
\hline Mean & & & \multirow{2}{*}{\multicolumn{2}{|c|}{$\begin{array}{c}1362 \cdot 4 \\
465 \cdot 7\end{array}$}} & & & \multicolumn{2}{|c|}{1365.4} & & & \multicolumn{2}{|c|}{$1241 \cdot 3$} & \multicolumn{2}{|c|}{$1267 \cdot 6$} \\
\hline SD & & & & & & & & & & & & & & \\
\hline \multicolumn{15}{|l|}{ Red meat $(\mathrm{g} / \mathrm{d})$} \\
\hline Mean & & & \multirow{2}{*}{\multicolumn{2}{|c|}{$\begin{array}{l}73.8 \\
64.0\end{array}$}} & & & \multicolumn{2}{|c|}{71.7} & & & \multicolumn{2}{|c|}{64.0} & \multirow{2}{*}{\multicolumn{2}{|c|}{$\begin{array}{l}64 \cdot 1 \\
53 \cdot 1\end{array}$}} \\
\hline SD & & & & & & & & & & & & & & \\
\hline$<43 \mathrm{~g} / \mathrm{d}$ & 23049.8 & $7 \cdot 0$ & 2963 & $36 \cdot 6$ & 165 & $42 \cdot 6$ & 61 & 44.9 & 22 & $47 \cdot 8$ & 22 & 41.5 & 44 & 44.4 \\
\hline$\geq 43 \mathrm{~g} / \mathrm{d}$ & 30976.8 & $7 \cdot 0$ & 4674 & 63.4 & 222 & 57.4 & 75 & $55 \cdot 1$ & 24 & $52 \cdot 2$ & 31 & 58.5 & 55 & 55.6 \\
\hline \multicolumn{15}{|c|}{ Vegetables and fruits $(\mathrm{g} / \mathrm{d})$} \\
\hline Mean & & & \multirow{2}{*}{\multicolumn{2}{|c|}{$\begin{array}{l}411.4 \\
259.5\end{array}$}} & \multirow{2}{*}{\multicolumn{2}{|c|}{$\begin{array}{l}406 \cdot 4 \\
245 \cdot 5\end{array}$}} & \multirow{2}{*}{\multicolumn{2}{|c|}{$\begin{array}{l}416.6 \\
261.8\end{array}$}} & \multirow{2}{*}{\multicolumn{2}{|c|}{$\begin{array}{l}415.5 \\
281.9\end{array}$}} & \multirow{2}{*}{\multicolumn{2}{|c|}{$\begin{array}{l}401.6 \\
236.5\end{array}$}} & \multirow{2}{*}{\multicolumn{2}{|c|}{$\begin{array}{l}408 \cdot 1 \\
257.3\end{array}$}} \\
\hline SD & & & & & & & & & & & & & & \\
\hline$<600 \mathrm{~g} / \mathrm{d}$ & $44186 \cdot 3$ & 6.9 & 6251 & $81 \cdot 8$ & 315 & 81.4 & 108 & 79.4 & 36 & $78 \cdot 3$ & 43 & $81 \cdot 1$ & 79 & 79.8 \\
\hline$\geq 600 \mathrm{~g} / \mathrm{d}$ & 9889.9 & $7 \cdot 1$ & 1386 & $18 \cdot 2$ & 72 & 18.6 & 28 & 20.6 & 10 & $21 \cdot 7$ & 10 & 18.9 & 20 & 20.2 \\
\hline \multicolumn{15}{|l|}{$\mathrm{Na}(\mathrm{mg} / \mathrm{d})$} \\
\hline Mean & & & \multirow{2}{*}{\multicolumn{2}{|c|}{$\begin{array}{l}4239 \cdot 8 \\
1682 \cdot 1\end{array}$}} & & & & & & & & & & \\
\hline SD & & & & & & & & & & & & & & \\
\hline$<4000 \mathrm{mg} / \mathrm{d}$ & 26679.9 & $7 \cdot 0$ & 3777 & 49.5 & 187 & $48 \cdot 3$ & 68 & $50 \cdot 0$ & 20 & 43.5 & 28 & $52 \cdot 8$ & 48 & 48.5 \\
\hline$\geq 4000 \mathrm{mg} / \mathrm{d}$ & $27396 \cdot 4$ & 7.0 & 3860 & 50.5 & 200 & $51 \cdot 7$ & 68 & 50.0 & 26 & 56.5 & 25 & $47 \cdot 2$ & 51 & 51.5 \\
\hline No. of dietary risk $f$ & & & & & & & & & & & & & & \\
\hline$\leq 2$ & $31202 \cdot 0$ & $7 \cdot 0$ & 4296 & $56 \cdot 3$ & 204 & 52.7 & 75 & 55.1 & 22 & $47 \cdot 8$ & 30 & 56.6 & 52 & 52.5 \\
\hline$\geq 3$ & 22824.6 & 6.9 & 3341 & 43.7 & 183 & $47 \cdot 3$ & 61 & 44.9 & 24 & $52 \cdot 2$ & 23 & 43.4 & 47 & 47.5 \\
\hline
\end{tabular}

*Tests of the association between the control and total cancer groups performed using the $\chi^{2}$ test (categorical variables) or a generalised linear model (continuous variables) adjusted for sex and age.

† Missing included no response or unwilling to respond.

$\ddagger$ All participants scored 1 point for each of the following dietary risk factors (high intakes of red meat and $\mathrm{Na}$, low intakes of vegetables and fruits, and obesity) at baseline: $\geq 43 \mathrm{~g}$ red meat/d; $<600 \mathrm{~g}$ vegetables and fruits/d; $\geq 4000 \mathrm{mg} \mathrm{Na} / \mathrm{d}$; obesity $\left(\mathrm{BMl} \geq 25 \mathrm{~kg} / \mathrm{m}^{2}\right)$. The number of dietary risk factors ranged from 0 to 4 points. 
Table 2. Hazard ratios (HR) for overall cancer according to dietary risk factors (Hazard ratios and $95 \%$ confidence intervals)

\begin{tabular}{|c|c|c|c|c|c|c|c|c|c|c|}
\hline & \multirow{2}{*}{\multicolumn{2}{|c|}{ Total $(n 8024)$}} & \multicolumn{4}{|c|}{ Sex } & \multicolumn{4}{|c|}{ Age } \\
\hline & & & \multicolumn{2}{|c|}{ Male ( $n$ 4402) } & \multicolumn{2}{|c|}{ Female ( $n$ 3622) } & \multicolumn{2}{|c|}{$<50$ years $(n 4506)$} & \multicolumn{2}{|c|}{$\geq 50$ years $(n 3518)$} \\
\hline & $\begin{array}{l}\text { Crude } \\
\mathrm{HR}^{\star} \quad 95 \% \mathrm{Cl}\end{array}$ & $\begin{array}{l}\text { Multivariate } \\
\mathrm{HR \dagger}\end{array}$ & $\begin{array}{c}\text { Crude } \\
\mathrm{HR}^{*}\end{array}$ & $\begin{array}{c}\text { Multivariate } \\
\text { HR† }\end{array}$ & $\begin{array}{c}\text { Crude } \\
\mathrm{HR}^{\star}\end{array}$ & $\begin{array}{c}\text { Multivariate } \\
\text { HR }+\end{array}$ & $\begin{array}{c}\text { Crude } \\
\mathrm{HR}^{*}\end{array}$ & $\begin{array}{c}\text { Multivariate } \\
\text { HR† }\end{array}$ & $\begin{array}{c}\text { Crude } \\
\mathrm{HR}^{*}\end{array}$ & $\begin{array}{c}\text { Multivariate } \\
\text { HR† }\end{array}$ \\
\hline Person-years & 54076 & & 29583 & & 24493 & & 30314 & & 23762 & \\
\hline Median & 7.0 & & 6.9 & & 7.0 & & 6.9 & & $7 \cdot 1$ & \\
\hline \multirow{2}{*}{\multicolumn{11}{|c|}{$\mathrm{BMI}\left(\mathrm{kg} / \mathrm{m}^{2}\right)$}} \\
\hline & & & & & & & & & & \\
\hline$<25$ & 1.00 & 1.00 & 1.00 & 1.00 & 1.00 & 1.00 & 1.00 & 1.00 & 1.00 & 1.00 \\
\hline$\underset{P}{\geq 25}$ & $\begin{array}{c}1.24 \underset{1.01,1.51}{0.0364} \\
0.04\end{array}$ & $\begin{array}{c}1.15 \underset{0.2554}{0.91,1.45} \\
0.54\end{array}$ & $\begin{array}{cc}1.03 & 0.79,1.33 \\
0.8559\end{array}$ & $\begin{array}{c}1.09 \underset{0.5629}{0.82,1.46} \\
0\end{array}$ & $\begin{array}{c}1 \cdot 60 \begin{array}{c}1.16,2 \cdot 19 \\
0.0039\end{array}\end{array}$ & $\begin{array}{c}1.48 \quad 0.98,2.24 \\
0.0623\end{array}$ & $\begin{array}{c}1.30 \\
0.95,1.77 \\
0.0965\end{array}$ & 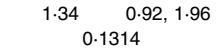 & $\begin{array}{cc}1.10 & 0.85,1.44 \\
0.4339\end{array}$ & $\begin{array}{c}1.08 \underset{0.79,1.46}{0.6343} \\
.\end{array}$ \\
\hline \multicolumn{11}{|l|}{ Red meat $(\mathrm{g} / \mathrm{d})$} \\
\hline$<43$ & 1.00 & 1.00 & 1.00 & 1.00 & 1.00 & 1.00 & 1.00 & 1.00 & 1.00 & 1.00 \\
\hline$\stackrel{\geq}{P}$ & $\begin{array}{ll}1.06 & 0.86,1 \cdot 30\end{array}$ & $1.23 \quad 0.96,1.57$ & $1.06 \quad 0.80,1.41$ & $1.41 \quad 1.02,1.94$ & $1.02 \quad 0.75,1.38$ & $0.97 \quad 0.66,1.44$ & $\begin{array}{ll}1.14 & 0.81,1.59\end{array}$ & $1.26 \quad 0.84,1.88$ & $1.18 \quad 0.90,1.53$ & $1.19 \quad 0.87,1.63$ \\
\hline \multicolumn{11}{|c|}{ Vegetables and fruits $(\mathrm{g} / \mathrm{d})$} \\
\hline$<600$ & 1.00 & 1.00 & 1.00 & 1.00 & 1.00 & 1.00 & 1.00 & 1.00 & 1.00 & 1.00 \\
\hline$\geq 600$ & $\begin{array}{cc}1.03 & 0.79,1.33 \\
0.8425 & \end{array}$ & $\begin{array}{c}1.02 \quad 0.75,1.37 \\
0.9217\end{array}$ & $\begin{array}{c}0.94 \quad 0.67,1.34 \\
0.7404\end{array}$ & $\begin{array}{l}0.93 \quad 0.63,1.38 \\
0.7229\end{array}$ & $\begin{array}{c}1.15 \quad 0.79,1.67 \\
0.4786\end{array}$ & $\begin{array}{c}1.14 \underset{0.71,1.85}{0.5864} \\
\end{array}$ & $\begin{array}{cc}1.05 \quad 0.69,1.60 \\
0.8076\end{array}$ & $\begin{array}{c}1.11 \quad 0.69,1.80 \\
0.6738\end{array}$ & $\begin{array}{c}0.92 \quad 0.66,1.27 \\
0.5947\end{array}$ & $\begin{array}{cc}0.95 & 0.65,1.40 \\
0.7923\end{array}$ \\
\hline \multicolumn{11}{|l|}{$\mathrm{Na}(\mathrm{mg} / \mathrm{d})$} \\
\hline$<4000$ & 1.00 & 1.00 & 1.00 & 1.00 & 1.00 & 1.00 & 1.00 & 1.00 & 1.00 & 1.00 \\
\hline$\stackrel{P}{P}$ & $\begin{array}{ll}1.04 & 0.86,1.27\end{array}$ & $\begin{array}{ll}1.18 & 0.91,1.52\end{array}$ & $\begin{array}{cc}0.97 & 0.75,1.27\end{array}$ & $\begin{array}{ll}1.28 & 0.93,1.76\end{array}$ & $\begin{array}{cc}1.09 & 0.80,1.49 \\
& 0.5707\end{array}$ & $1.01 \quad 0.66,1.56$ & $\begin{array}{ll}1.13 & 0.83,1.55 \\
0.4236\end{array}$ & $\begin{array}{lr}1.36 & 0.92,2.02\end{array}$ & $\begin{array}{cc}1.02 & 0.78,1.32 \\
0.9136\end{array}$ & $\begin{array}{lll}1.06 & 0.76,1.49\end{array}$ \\
\hline \multicolumn{3}{|c|}{ No. of dietary risk factors $\ddagger$} & & & & & & & & \\
\hline$\leq 2$ & & 1.00 & 1.00 & 1.00 & 1.00 & 1.00 & 1.00 & 1.00 & 1.00 & 1.00 \\
\hline$\geq 3$ & $1.24 \quad 1.01,1 \cdot 51$ & $1.26 \quad 0.99,1.60$ & $1.05 \quad 0.80,1 \cdot 36$ & $\begin{array}{ll}1.31 & 0.97,1.77\end{array}$ & $1.52 \quad 1.11,2.08$ & $1.23 \quad 0.81,1.88$ & $1.44 \quad 1.05,1.96$ & $\begin{array}{ll}1.46 & 0.99,2.16\end{array}$ & $1.19 \quad 0.91,1.55$ & $1.17 \quad 0.86,1.61$ \\
\hline$P$ & 0.0372 & 0.0658 & 0.7482 & & 0.0091 & & 0.0218 & 0.0545 & 0.2035 & 0.3201 \\
\hline
\end{tabular}

${ }^{\star}$ Estimated using the Cox proportional hazards regression model.

†Estimated using the Cox proportional hazards regression model, adjusted for age, sex, energy intake, BMI, physical activity, smoking, alcohol use, income, education and marital status.

¥All participants scored 1 point for each of the following dietary risk factors (high intakes of red meat and $\mathrm{Na}$, low intakes of vegetables and fruits, and obesity) at baseline: $\geq 43 \mathrm{~g}$ red meat/d; $<600 \mathrm{~g}$ vegetables and fruits/d; $\geq 4000 \mathrm{mg} \mathrm{Na} / \mathrm{d}$; obesity $\left(\mathrm{BMI} \geq 25 \mathrm{~kg} / \mathrm{m}^{2}\right)$. The number of dietary risk factors ranged from 0 to 4 points. 
Table 3. Hazard ratios (HR) for major cancers according to dietary risk factors

(Hazard ratios and $95 \%$ confidence intervals)

\begin{tabular}{|c|c|c|c|c|c|c|c|c|c|c|c|c|c|c|c|c|}
\hline & \multicolumn{4}{|c|}{ Thyroid cancer ( $n$ 136) } & \multicolumn{4}{|c|}{ Gastric cancer ( $n$ 46) } & \multicolumn{4}{|c|}{ Colorectal cancer ( $n$ 53) } & \multicolumn{4}{|c|}{ Gastric and colorectal cancers ( $n$ 99) } \\
\hline & $\begin{array}{c}\text { Crude } \\
\text { HR }^{*}\end{array}$ & $95 \% \mathrm{Cl}$ & $\begin{array}{c}\text { Multivaria } \\
\text { HR† }\end{array}$ & $95 \% \mathrm{Cl}$ & $\begin{array}{l}\text { Crude } \\
\mathrm{HR}^{*}\end{array}$ & $95 \% \mathrm{Cl}$ & $\begin{array}{c}\text { Multivaria } \\
\text { HR† }\end{array}$ & $95 \% \mathrm{Cl}$ & $\begin{array}{c}\text { Crude } \\
\text { HR }^{*}\end{array}$ & $95 \% \mathrm{Cl}$ & $\begin{array}{c}\text { Multivaria } \\
\text { HR† }\end{array}$ & $95 \% \mathrm{Cl}$ & $\begin{array}{l}\text { Crude } \\
\mathrm{HR}^{*}\end{array}$ & $95 \% \mathrm{Cl}$ & $\begin{array}{c}\text { Multivariate } \\
\text { HR } \dagger\end{array}$ & $95 \% \mathrm{Cl}$ \\
\hline Person-years & \multicolumn{2}{|r|}{223} & \multicolumn{6}{|c|}{68} & \multicolumn{2}{|r|}{53} & \multicolumn{6}{|c|}{121} \\
\hline Median & \multirow{2}{*}{\multicolumn{2}{|c|}{$\begin{array}{c}0.7 \\
0.0-8 \cdot 3\end{array}$}} & & & & 0.3 & & & & 0.1 & & & & $0 \cdot 1$ & & \\
\hline Range & & & \multirow{2}{*}{\multicolumn{2}{|c|}{1.00}} & \multicolumn{2}{|c|}{$\begin{array}{c}0.3 \\
0.0-8 \cdot 4\end{array}$} & \multirow{2}{*}{\multicolumn{2}{|c|}{1.00}} & \multicolumn{2}{|c|}{$0.0-6.0$} & \multicolumn{6}{|c|}{$0.0-8.4$} \\
\hline $\begin{array}{l}\mathrm{BMI}\left(\mathrm{kg} / \mathrm{m}^{2}\right) \\
<25 \mathrm{~kg} / \mathrm{m}^{2}\end{array}$ & \multicolumn{2}{|c|}{1.00} & & & & 1.00 & & & & 1.00 & \multicolumn{2}{|r|}{1.00} & & 1.00 & \multicolumn{2}{|c|}{1.00} \\
\hline$\geq 25 \mathrm{~kg} / \mathrm{m}^{2}$ & 1.34 & $0.96,1.87$ & 1.56 & $1 \cdot 05,2 \cdot 31$ & 1.96 & $1.09,3.51$ & 1.84 & $0.90,3.76$ & 0.85 & $0.48,1.48$ & 0.64 & $0.32,1.26$ & 0.88 & $0.59,1.31$ & 0.76 & $0.43,1.33$ \\
\hline & \multicolumn{2}{|c|}{0.0898} & \multirow{2}{*}{\multicolumn{2}{|c|}{0.0273}} & \multirow{2}{*}{\multicolumn{2}{|c|}{0.0239}} & \multirow{2}{*}{\multicolumn{2}{|c|}{0.0941}} & \multirow{2}{*}{\multicolumn{2}{|c|}{ NS }} & \multicolumn{2}{|r|}{ NS } & \multirow{2}{*}{\multicolumn{2}{|c|}{ NS }} & \multicolumn{2}{|c|}{ NS } \\
\hline \multicolumn{7}{|l|}{ Red meats $(\mathrm{g} / \mathrm{d})$} & & & & & & & & & & \\
\hline$<43$ & \multicolumn{2}{|c|}{1.00} & \multicolumn{2}{|c|}{1.00} & \multicolumn{2}{|r|}{1.00} & \multicolumn{2}{|c|}{1.00} & \multicolumn{2}{|r|}{1.00} & & 1.00 & & 1.00 & & \\
\hline$P 43$ & 0.91 & $0.65,1 \cdot 28$ & 0.91 & $S^{0.61,1 \cdot 36}$ & 0.76 & $0.42,1.35$ & $1 \cdot 16$ & $0.56,2 \cdot 41$ & 1.05 & $\begin{array}{l}0.60,1.82 \\
N S\end{array}$ & 1.31 & $\mathrm{NS}^{0.60,2 \cdot 61}$ & $1 \cdot 11$ & $0.74,1.65$ & 1.48 & $0.83,2.64$ \\
\hline Vegetables and & ruits $(\mathrm{g} / \mathrm{c}$ & & & & & & & & & & & & & & & \\
\hline$<600$ & & 1.00 & & 00 & & 1.00 & & 00 & & 1.00 & & 1.00 & & 1.00 & & \\
\hline$P_{P}^{\geq 600}$ & 0.86 & $\begin{array}{l}0.57,1.30 \\
\text { NS }\end{array}$ & 0.87 & $S^{0.54,1.42}$ & 0.80 & $\begin{array}{l}0.40,1.62 \\
N S\end{array}$ & 0.83 & $S^{0.35,1.98}$ & 0.96 & $\begin{array}{l}0.48,1.90 \\
\text { NS }\end{array}$ & 0.85 & NS ${ }^{0.38,1.92}$ & 0.91 & $\begin{array}{l}0.55,1.51 \\
N S\end{array}$ & 0.72 & $0.38,1.36$ \\
\hline $\mathrm{Na}(\mathrm{mg} / \mathrm{d})$ & & & & & & & & & & & & & & & & \\
\hline$<4000$ & & 1.00 & & 00 & & 1.00 & & 00 & & 1.00 & & 1.00 & & 1.00 & & \\
\hline$\stackrel{\geq}{P} 4000$ & 0.98 & $\begin{array}{l}0.70,1.37 \\
N S\end{array}$ & $1 \cdot 11$ & 15 & $1 \cdot 27$ & $\begin{array}{l}0.71,2 \cdot 28 \\
N S\end{array}$ & $2 \cdot 34$ & $367.05,5 \cdot 19$ & 0.87 & $\begin{array}{l}0.51,1.50 \\
\text { NS }\end{array}$ & 1.52 & $\begin{array}{l}0.75,3.08 \\
0.2446\end{array}$ & 1.57 & $\begin{array}{l}1.04,2.35 \\
0.0303\end{array}$ & ${ }^{4 \cdot 28}<c$ & $\begin{array}{l}2 \cdot 11,8 \cdot 72 \\
001\end{array}$ \\
\hline No. of dietary ris & factors & & & & & & & & & & & & & & & \\
\hline$\leq 2$ & & 1.00 & & 00 & & 1.00 & & 00 & & 1.00 & 1.00 & & & 1.00 & & \\
\hline $\begin{array}{l}\geq 3 \\
P\end{array}$ & 1.08 & $\begin{array}{l}0.77,1.51 \\
\text { NS }\end{array}$ & $1 \cdot 16$ & IS & 1.53 & $\begin{array}{l}0.86,2 \cdot 73 \\
\text { NS }\end{array}$ & 2.07 & $\begin{array}{l}0.99,4.33 \\
533\end{array}$ & 0.99 & $\begin{array}{l}0.57,1.70 \\
\text { NS }\end{array}$ & 1.04 & NS ${ }^{0.53,2.04}$ & $1 \cdot 11$ & $\begin{array}{l}0.74,1.65 \\
\text { NS }\end{array}$ & ${ }^{1.87} 0$ & $8^{1.03,3 \cdot 37}$ \\
\hline
\end{tabular}

*Estimated using the Cox proportional hazards regression model.

†Estimated using the Cox proportional hazards regression model, adjusted for age, sex, energy intake, BMI, physical activity, smoking, alcohol use, income, education and marital status.

FAll participants scored 1 point for each of the following dietary risk factors (high intakes of red meat and Na, low intakes of vegetables and fruits, and obesity) at baseline: $\geq 43 \mathrm{~g}$ red meat/d; $<600 \mathrm{~g}$ vegetables and fruits/d; $\geq 4000 \mathrm{mg} \mathrm{Na} / \mathrm{d}$; obesity $\left(\mathrm{BMI} \geq 25 \mathrm{~kg} / \mathrm{m}^{2}\right.$ ). The number of dietary risk factors ranged from 0 to 4 points. 


\section{Discussion}

This is the first prospective cohort study using the openended dietary assessment method to estimate the association between dietary risk factors and cancer incidence among Koreans, and found that dietary risk factors such as red meat and $\mathrm{Na}$ intakes were significantly positively associated with cancer risk in a Korean population.

People are living longer than ever before, and the incidence of cancer has been increased with ageing ${ }^{(39-41)}$. In the present study, overall cancer risk was positively associated with age and significantly higher in individuals older than or equal to 50 years compared with those younger than 50 years of age (HR 1.80, 95\% CI 1.41, 2.31; $P<0.0001$; data not shown).

We also found that obesity (BMI $\geq 25 \mathrm{~kg} / \mathrm{m}^{2}$ ) tended to increase the risk of overall cancer in women, which was positively associated with the risk of thyroid cancer. Many studies have reported that overweight $\left(25 \leq \mathrm{BMI}<30 \mathrm{~kg} / \mathrm{m}^{2}\right)$ and obese (BMI $\geq 30 \mathrm{~kg} / \mathrm{m}^{2}$ ) individuals have an increased risk of various cancers, primarily in Western populations, but not among Asians ${ }^{(7,17,18,20,21)}$. In contrast, one 14-year prospective cohort study conducted by the National Health Insurance Corporation in Koreans has shown that obesity was significantly associated with an increased risk of cancer ${ }^{(19)}$. The obesity criteria (BMI $\geq 30 \mathrm{~kg} / \mathrm{m}^{2}$ ) used in the studies for the Western population were different from those for Asians (BMI $\geq 25 \mathrm{~kg} / \mathrm{m}^{2}$ ). Because Asian populations generally have a higher percentage of body fat compared with the Westerners at the same $\mathrm{BMI}^{(36)}$, our findings support that overweight $\left(23 \leq \mathrm{BMI}<25 \mathrm{~kg} / \mathrm{m}^{2}\right)$ as well as obesity $\left(\mathrm{BMI} \geq 25 \mathrm{~kg} / \mathrm{m}^{2}\right)$ can also contribute to the risk of cancer in Korean populations.

Most previous studies have reported that red meat consumption is an important risk factor for cancer at several sites $^{(7-13)}$. In the present study, a significant positive association between red meat intake and overall cancer risk was observed only in men. We speculated the reason why the risk of meat intake increased cancer risk only in men, and found that the meat intake level was significantly higher in men than in women. The mean intake of red meat and the percentage of individuals who consumed at least $43 \mathrm{~g}$ red meat daily were significantly higher in men $(85.3 \mathrm{~g}$ and $68.1 \%$, respectively) than in women $(59.5 \mathrm{~g}$ and $52.9 \%$, respectively) ( $P<0 \cdot 0001$, data not shown). In contrast, the effects of red meat type, fresh and processed red meat on the risk of cancer were analysed. We found that fresh red meat was not associated with cancer risk, while processed red meat intake was positively associated with cancer risk in men (HR 1.05, $95 \%$ CI 1.00, 1.92; $P=0.0343$ ) and in individuals older or equal to 50 years of age (HR 1.07, $95 \%$ CI 1.02, 1.13; $P=0.0068$ ) (data not shown).

These results suggested that meat intake, especially processed meat intake, of men or people older than or equal to 50 years of age should be cautioned for cancer prevention.

To our knowledge, many previous studies have examined the combined effects of lifestyle-related risk factors, including diet, on cancer risk ${ }^{(3,7,42-48)}$, but not many studies have examined the effects of combined dietary risk factors on cancer risk in Asia, especially in Korea. Notably, we found that the combined effects of dietary risk factors on overall cancer risk were greater than the individual effects. These results provide new evidence that dietary risk factors, including red meat and $\mathrm{Na}$ intakes, and obesity, may contribute to increased cancer risk in a population when combined, as in those who are experiencing a dietary transition from a traditional diet composed of mainly rice and vegetables to a Westernised diet.

Dietary risk factors are known to be distinct according to the cancer site. Many previous studies have suggested that the heavy consumption of $\mathrm{Na}$ is a risk factor for gastric cancer $^{(3,15,16)}$, red meat consumption is for colorectal cancer $^{(7-10)}$ and obesity is for thyroid cancer ${ }^{(3,49,50)}$. We also observed that the risk of gastric cancer increased with $\mathrm{Na}$ intake and thyroid cancer risk was increased with obesity.

Previous studies have suggested that thyroid cancer was linked to iodine intake ${ }^{(3,51)}$. We analysed the association between thyroid cancer and seaweeds as the main sources of iodine, because there was no available iodine database for common foods in Korea. Seaweed intake was higher in the thyroid cancer group $(4 \cdot 28(\mathrm{SD} 12 \cdot 0) \mathrm{g} / \mathrm{d})$ than in the control group $(4 \cdot 19(\mathrm{SD} 13.6) \mathrm{g} / \mathrm{d})$, but there was no statistically significant association between seaweed consumption and the risk of thyroid cancer (data not shown). Further studies are needed to clarify the association between the risk of thyroid cancer and iodine or seaweed intake.

Both gastric cancer and colorectal cancer risks were not associated with the number of dietary risk factors, but the risk of gastrointestinal cancers including both sites was positively associated with the number of dietary risk factors. The reason may be due to the increased number of cancer patients to get sufficient analysis power.

The strength of the present study was its large prospective cohort design, which enabled us to investigate the association between diet and cancer risk in Korea. Second, the present study used $3 \mathrm{~d}$ dietary records, which can accurately measure the dietary intake of subjects, and recall bias and reverse causation were minimised by the assessment of diet before cancer diagnosis. Moreover, since all kinds of meat consumed by the subjects were measured, the amount of red meat intake must be accurate to provide statistical power to detect associations.

The present study also had certain limitations. First, the mean follow-up period of the cohort in the present study was 6.7 years, which might be insufficient to identify a large number of incident cancer cases for statistical power. The small number of cancer cases may limit an adequate assessment of the relationship between diet and cancer, result in low statistical power to identify the association and cause potential problems according to the multiple testing. Second, the present study was conducted with self-motivated participants in a hospital setting, and thus might be susceptible to selection bias. The participants in the present study were limited to those individuals who could afford the expenses of the cancer screening programme. Third, the dietary risk factors used in the present study were subjectively selected according to the WCRF/AICR recommendation; because dietary guidelines have not been developed to prevent cancer in Koreans and few studies have been performed to identify dietary risk factors in Koreans, important dietary risk factors were not 
included in the analysis. Fourth, dietary information might not reflect an individual's usual intake because we used $3 \mathrm{~d}$ food records.

In conclusion, we observed a positive association between red meat and $\mathrm{Na}$ intakes and cancer risk in the Korean population. The findings can be applied to develop and evaluate a cancer control programme for Koreans, although further studies are needed to clarify dietary risk factors for cancer at specific sites among Koreans.

\section{Supplementary material}

To view supplementary material for this article, please visit http://dx.doi.org/10·1017/S0007114514000683

\section{Acknowledgements}

The authors are indebted to the dietitians at the National Cancer Center for data collection.

The present study was partially supported by the National Cancer Center of the Republic of Korea (NCC0910220).

The authors' responsibilities were as follows: G.-A. W., J. K. and H. J. designed the research; Y.-A. C., H.-h. K., K.A. R., and M.-K. Y. conducted the research; G.-A. W. analysed the data and wrote the manuscript; K.-W. J. helped to analyse and interpret the data; Y.-A. K. and J.-H. L. helped to draft the manuscript; G.-A. W. and H. J. had primary responsibility for the final content. All authors read and approved the final manuscript.

None of the authors had a conflict of interest.

\section{References}

1. Jung KW, Park S, Kong HJ, et al. (2010) Cancer statistics in Korea: incidence, mortality and survival in 2006-2007. J Korean Med Sci 25, 1113-1121.

2. Jung KW, Won YJ, Kong HJ, et al. (2013) Cancer statistics in Korea: incidence, mortality, survival and prevalence in 2010. Cancer Res Treat 45, 1-14.

3. World Cancer Research Fund and American Institute for Cancer Research (2007) Food, Nutrition, Physical Activity, and the Prevention of Cancer: A Global Perspective. Washington, DC: AICR.

4. Zhang J, Dhakal IB, Zhao Z, et al. (2012) Trends in mortality from cancers of the breast, colon, prostate, esophagus, and stomach in East Asia: role of nutrition transition. Eur J Cancer Prev 21, 480-489.

5. Magalhães B, Bastos J \& Lunet N (2011) Dietary patterns and colorectal cancer: a case-control study from Portugal. Eur J Cancer Prev 20, 389-395.

6. Egeberg R, Olsen A, Autrup H, et al. (2008) Meat consumption, $N$-acetyl transferase 1 and 2 polymorphism and risk of breast cancer in Danish postmenopausal women. Eur $J$ Cancer Prev 17, 39-47.

7. Key TJ, Schatzkin A, Willett WC, et al. (2004) Diet, nutrition and the prevention of cancer. Public Health Nutr 7, $187-200$

8. Norat T, Bingham S, Ferrari P, et al. (2005) Meat, fish, and colorectal cancer risk: the European Prospective Investigation into Cancer and Nutrition. J Natl Cancer Inst 97, 906-916.
9. Cross AJ, Leitzmann MF, Gail MH, et al. (2007) A prospective study of red and processed meat intake in relation to cancer risk. PLoS Med 4, e325.

10. Flood A, Rastogi T, Wirfalt E, et al. (2008) Dietary patterns as identified by factor analysis and colorectal cancer among middle-aged Americans. Am J Clin Nutr 88, 176-184.

11. Larsson SC \& Wolk A (2012) Red and processed meat consumption and risk of pancreatic cancer: meta-analysis of prospective studies. Br J Cancer 106, 603-607.

12. Keszei AP, Schouten LJ, Goldbohm RA, et al. (2012) Red and processed meat consumption and the risk of esophageal and gastric cancer subtypes in The Netherlands Cohort Study. Ann Oncol 23, 2319-2326.

13. Fung TT, Hu FB, Holmes MD, et al. (2005) Dietary patterns and the risk of postmenopausal breast cancer. Int J Cancer 116, 116-121.

14. Divisi D, Di Tommaso S, Salvemini S, et al. (2006) Diet and cancer. Acta Biomed 77, 118-123.

15. Lee JK, Park BJ, Yoo KY, et al. (1995) Dietary factors and stomach cancer: a case-control study in Korea. Int J Epidemiol 24, 33-41.

16. Kim J, Park S \& Nam BH (2010) Gastric cancer and salt preference: a population-based cohort study in Korea. Am J Clin Nutr 91, 1289-1293.

17. Percik R \& Stumvoll M (2009) Obesity and cancer. Exp Clin Endocrinol Diabetes 117, 563-566.

18. Pischon T, Lahmann PH, Boeing $\mathrm{H}$, et al. (2006) Body size and risk of colon and rectal cancer in the European Prospective Investigation Into Cancer and Nutrition (EPIC). J Natl Cancer Inst 98, 920-931.

19. Jee SH, Yun JE, Park EJ, et al. (2008) Body mass index and cancer risk in Korean men and women. Int J Cancer 123, 1892-1896.

20. Martinez ME, Giovannucci E, Spiegelman D, et al. (1997) Leisure-time physical activity, body size, and colon cancer in women. Nurses' Health Study Research Group. J Natl Cancer Inst 89, 948-955.

21. Bergstrom A, Pisani P, Tenet V, et al. (2001) Overweight as an avoidable cause of cancer in Europe. Int J Cancer 91, 421-430.

22. Samanic C, Chow WH, Gridley G, et al. (2006) Relation of body mass index to cancer risk in 362,552 Swedish men. Cancer Causes Control 17, 901-909.

23. Oh SW, Yoon YS \& Shin SA (2005) Effects of excess weight on cancer incidences depending on cancer sites and histologic findings among men: Korea National Health Insurance Corporation Study. J Clin Oncol 23, 4742-4754.

24. Doll R \& Peto R (1981) The causes of cancer: quantitative estimates of avoidable risks of cancer in the United States today. J Natl Cancer Inst 66, 1191-1308.

25. Kim S, Moon S \& Popkin BM (2000) The nutrition transition in South Korea. Am J Clin Nutr 71, 44-53.

26. Lee SK \& Sobal J (2003) Socio-economic, dietary, activity, nutrition and body weight transitions in South Korea. Public Health Nutr 6, 665-674.

27. Lee MJ, Popkin BM \& Kim S (2002) The unique aspects of the nutrition transition in South Korea: the retention of healthful elements in their traditional diet. Public Health Nutr 5, 197-203.

28. Song $\mathrm{Y}$, Joung $\mathrm{H}$, Engelhardt $\mathrm{K}$, et al. (2005) Traditional $v$. modified dietary patterns and their influence on adolescents' nutritional profile. Br J Nutr 93, 943-949.

29. Kang M, Joung H, Lim JH, et al. (2011) Secular trend in dietary patterns in a Korean adult population, using the 1998, 2001, and 2005 Korean National Health and Nutrition Examination Survey. Korean J Nutr 44, 152-161. 
30. Kwon JH, Shim JE, Park MK, et al. (2009) Evaluation of fruits and vegetables intake for prevention of chronic disease in Korean adults aged 30 years and over: using the Third Korea National Health and Nutrition Examination Survey (KNHANES III), 2005. Korean J Nutr 42, 146-157.

31. Suh SW, Koo BK, Jeon SH, et al. (2005) Analysis of dietary risk factors of the colorectal cancer patients in Daegu Kyungpook area, Korea (I) - a study on lifestyle and eating behaviors of the colorectal cancer patients. Korean J Nutr 38, 125-143.

32. Lee EJ, Suh SW, Lee WK, et al. (2007) Reproductive factor and food intake pattern influencing on the breast cancer risk in Daegu Gyungbuk area, Korea. Korean J Nutr $\mathbf{4 0}$, 334-346.

33. Oh SY, Lee JH \& Kim HJ (2004) Analyses on the associations of dietary patterns with colon cancer risk. Korean J Nutr 37 , $550-556$.

34. Kim J, Kim DH, Lee BH, et al. (2009) Folate intake and the risk of colorectal cancer in a Korean population. Eur J Clin Nutr 63, 1057-1064.

35. World Health Organization (1994) International Classification of Diseases and Related Health Problems, 10th ed. Geneva, Switzerland: WHO.

36. WHO Expert Consultation (2004) Appropriate body-mass index for Asian populations and its implications for policy and intervention strategies. Lancet 363, 157-163.

37. Wen CP, David Cheng TY, Tsai SP, et al. (2009) Are Asians at greater mortality risks for being overweight than Caucasians? Redefining obesity for Asians. Public Health Nutr 12, 497-506.

38. Ministry of Health and Welfare (2011) Annual Report of Cancer Statistics in Korea in 2009. Seoul: Korean Government Publishing Service.

39. Anisimov VN, Sikora E \& Pawelec G (2009) Relationships between cancer and aging: a multilevel approach. Biogerontology 10, 323-338.

40. Franceschi S \& La Vecchia C (2001) Cancer epidemiology in the elderly. Crit Rev Oncol Hematol 39, 219-226.

41. Schmidlin K, Spoerri A, Egger M, et al. (2012) Cancer, a disease of aging (part 2) - risk factors for older adult cancer mortality in Switzerland 1991-2008. Swiss Med Wkly 142, w13607.

42. Gonzalez CA (2006) Nutrition and cancer: the current epidemiological evidence. Br J Nutr 96, Suppl. 1, 42S-45S.

43. van Dam RM, Li T, Spiegelman D, et al. (2008) Combined impact of lifestyle factors on mortality: prospective cohort study in US women. BMJ 337, a1440.

44. Huxley RR, Ansary-Moghaddam A, Clifton P, et al. (2009) The impact of dietary and lifestyle risk factors on risk of colorectal cancer: a quantitative overview of the epidemiological evidence. Int J Cancer 125, 171-180.

45. Ford ES, Bergmann MM, Kroger J, et al. (2009) Healthy living is the best revenge: findings from the European Prospective Investigation Into Cancer and Nutrition-Potsdam Study. Arch Intern Med 169, 1355-1362.

46. Kirkegaard H, Johnsen NF, Christensen J, et al. (2010) Association of adherence to lifestyle recommendations and risk of colorectal cancer: a prospective Danish cohort study. BMJ 341, c5504.

47. Kushi LH, Doyle C, McCullough M, et al. (2012) American Cancer Society Guidelines on nutrition and physical activity for cancer prevention: reducing the risk of cancer with healthy food choices and physical activity. CA Cancer J Clin 62, 30-67.

48. Nahleh Z, Bhatti NS \& Mal M (2011) How to reduce your cancer risk: mechanisms and myths. Int J Gen Med 4, $277-287$.

49. Engeland A, Tretli S, Akslen LA, et al. (2006) Body size and thyroid cancer in two million Norwegian men and women. Br J Cancer 95, 366-370.

50. Kitahara CM, Platz EA, Freeman LE, et al. (2011) Obesity and thyroid cancer risk among U.S. men and women: a pooled analysis of five prospective studies. Cancer Epidemiol Biomarkers Prev 20, 464-472.

51. Michikawa T, Inoue M, Shimazu T, et al. (2012) Seaweed consumption and the risk of thyroid cancer in women: the Japan Public Health Center-based Prospective Study. Eur J Cancer Prev 21, 254-260. 